\title{
Conformational Analysis of Stereoisomers of Triphenylheptane by Depolarized Rayleigh Scattering
}

\author{
Georges Fourche, Bruno JAsse, ${ }^{*}$ and Pierre Maelstaf \\ Centre de Recherches Paul Pascal, Domaine Universitaire, \\ 33405 Talence, France. \\ * Laboratoire de Physico-Chimie Structurale et Macromoléculaire, \\ Associé au CNRS, 10 rue Vauquelin, \\ 75231 Paris Cedex 05, France.
}

(Received January 13, 1977)

\begin{abstract}
S: Average molecular optical anisotropies $\left\langle\gamma^{2}\right\rangle$ of the three stereoisomers of 2,4,6-triphenylheptane were measured by depolarized Rayleigh scattering. Analysis of these results led to the determination of the relative stability among the various conformational structures of these isomers.

KEY WORDS Optical Anisotropy / Rayleigh Scattering / Local Conformation / Triphenylheptane /
\end{abstract}

In a previous work ${ }^{1,2}$ we studied by depolarized Rayleigh scattering (DRS) ${ }^{3}$ the stereoregularity of polystyrene (PS) chains in solution. The quantitative interpretation ${ }^{2}$ of our results led to the study of short range interactions in model molecules of PS, the meso and racemic 2,4-diphenylpentane (DPP). This paper is devoted to the study by depolarized Rayleigh scattering of conformational structure of three stereoisomers of 2,4,6-triphenylheptane (TPH) which are the higher members of the series. These results will be compared with those already published $^{4,8}$ elsewhere. The experimental and theoretical basis for the method used will not be developed here but may be found in ref $1-6$.

\section{EXPERIMENTAL}

The synthesis of 2,4,6-triphenylheptane was carried out ${ }^{7}$ by condensing cumyl magnesium bromide with 1,3-diphenyl-1-butanone. The different configurational isomers were at the first step separated by preparative gas chromatography using a Thomson SRTI model THN 102 with a stationary phase of the silicon SE-30 type. The purity of the heterotactic isomer was estimated at about 95\% and that of isotactic and syndiotactic isomers at $99 \%$. On completion of this work we noted that the heterotactic isomer was colorless but, that other isomers had a slightly yellow color. This coloration which may be introduced by eventual fluorescent molecules was very inconvenient for subsequent measurement of Rayleigh scattering. Also in the second step, we carried out a repurification of the compounds in question. Thus, isotactic and syndiotactic TPH were dissolved in cyclohexane and the solutions obtained were filtered on active coal. The stereoisomers were at last recovered by evaporation under vacuum of the solvent. Each compound so obtained was a transparent colorless liquid.

\section{MOLECULAR OPTICAL ANISOTROPY AND CONFORMATIONAL ANALYSIS}

The average molecular optical anisotropy $\left\langle\gamma^{2}\right\rangle^{3}$ of TPH in cyclohexane solution at $25^{\circ} \mathrm{C}$ was determined* by measuring the scattered depolarized intensity from the solutions at a wavelengh of $5460 \AA$. The technique used has been previously described. ${ }^{3,1}$ The accuracy of $\left\langle\gamma^{2}\right\rangle$ is

* In practice the intensity measurements are carried out by comparison with a standard liquid, cyclohexane, for which these has been determined at a wavelength of $5460 \AA$ a scattered depolarized intensity per unit volume and unit of incident photometric illumination of $23,15 \cdot 10^{-8} \mathrm{~cm}^{-1}$. 
Table I. Experimental and theoreticala molecular optical anisotropies (cyclohexane solution) of stereoisomers of TPH at $25^{\circ} \mathrm{C}$

$$
\left\langle r^{2}\right\rangle, \AA^{6}
$$

\begin{tabular}{lccc}
\hline Stereoisomers & Experimental & Calculated & $\begin{array}{c}\text { Deviation, } \\
\%\end{array}$ \\
\hline Hetero TPH & 229 & 229.5 & 0 \\
Syndio TPH & 243 & 253.5 & 4.3 \\
Iso TPH & 211 & 203.3 & 3.7 \\
\hline
\end{tabular}

a Calculated according to the parameter of ref 2 .

about 3 or $4 \%$. Experimental results are indicated in Table I with the theoretical molecular optical anisotropies calculated according to the rotational isomer model and the method developed by Tonelli, Abe, and Flory ${ }^{5}$ for vinyl polymers. At this point, it is necessary to recall that the theoretical molecular optical anisotropy of a stereoirregular chain with a lateral substituent $R$ is a function of various parameters, in particular, the statistical weights $\eta$ and $\tau,{ }^{*}$ the rotational angle $\chi$ of the $\mathrm{R}$ group about the $\mathrm{CH}-\mathrm{R}$ bond and of the probability $w_{\text {syn }}$ for a syndiotactic placement of the stereoregular unit relative to the preceding unit. Statistical weights $\eta$ and $\tau^{*}$ take into account the interactions of a $\mathrm{CH}$ group with $\mathrm{R}$ and the constraints imposed on rotations around $\mathrm{CH}-\mathrm{R}$ bond respectively if $\mathrm{R}$ is an articulated substituent.

The theoretical $\left\langle r^{2}\right\rangle$ of the three stereoisomers of TPH (see Table I) has been calculated according to the eq 9 and the method of the ref 2 with the corresponding choice of parameters. In particular we have used $\eta=1.48, \tau^{*}=1$ and $\chi=20^{\circ}$; values determined from our previous experimental studies. ${ }^{2}$ For heterotactic stereoisomer the theoretical molecular optical anisotropy has been calculated in the following manner: ${ }^{* *}$ if $\left\langle\gamma^{2}\right\rangle_{\text {iso }}$ is the average molecular optical anisotropy of a $\mathrm{mm}$ triad of the stereoirregular chain, and $\left\langle\gamma^{2}\right\rangle_{\mathrm{syn}}$ that of $r r$ triad and $\left\langle\gamma^{2}\right\rangle_{\text {hetero }}$ that of $m r$ or $r m$ triad, one can write in the hypothesis of a Bernoullian propagation process and with $^{2} w_{\text {syn }}=0.50$ :

\footnotetext{
** $\left\langle\gamma^{2}\right\rangle_{\text {hetero }}$ is calculated by an indirect method in order to use numerical results immediately available from our previous study. ${ }^{2}$
}

$$
\frac{2\left\langle\gamma^{2}\right\rangle_{\text {hetero }}+\left\langle\gamma^{2}\right\rangle_{\text {iso }}+\left\langle\gamma^{2}\right\rangle_{\text {sgn }}}{4}=\left\langle\gamma^{2}\right\rangle_{\text {atac }}
$$

In this expression, $\left\langle\gamma^{2}\right\rangle_{\text {atac }}$ is the calculated molecular optical anisotropy equal to $228.96 \dot{\AA}^{6}$ of the "atactic" trimer molecule. The above equation allows us to obtain $\left\langle\gamma^{2}\right\rangle_{\text {hetero }}$.

Examination of Table I shows an excellent agreement between the experimental and theoretical values. Thus, deviations between observed and calculated values of $\left\langle\gamma^{2}\right\rangle$ are $0,4.3$ and $3.7 \%$ for heterotactic, syndiotactic, and isotactic molecules, respectively. This agreement between experiment and theory is an additional confirmation of the correct choice previously ${ }^{2}$ made with the aid of 2,4-diphenylpentane using the different conformational parameters which give short range interactions in these molecules. In particular, it is interesting to note that we have verified in our previous study ${ }^{2}$ on PS chains that molecular optical anisotropy of isotactic TPH is for the most part independent of the factor $\eta$ but very sensitive to the angle $\chi$.

Therefore it can be deduced that the value of $\chi=20^{\circ}$ is also correct for our experimental $\left\langle\gamma^{2}\right\rangle$ measurement ( 3 or $4 \%$ ). This parameter $\chi$ may be indeed useful in the study of chromicity phenomena of these molecules and it seems that untill now, only DRS has given an experimental value of $\chi$.

Now we must make a comparison of the experimental data, obtained by DRS and NMR, with the relative stabilities of different con-

Table II. Values at $25^{\circ} \mathrm{C}$ of the statistical weight

\begin{tabular}{|c|c|c|c|c|c|}
\hline $\begin{array}{l}\text { Stereo- } \\
\text { isomers }\end{array}$ & $\begin{array}{l}\text { Con- } \\
\text { formers }\end{array}$ & $\begin{array}{c}\text { Statist. } \\
\text { weight- } \\
\text { equil. } \\
\text { compos. }\end{array}$ & $\begin{array}{l}\Delta G, \\
\mathrm{cal}\end{array}$ & $\begin{array}{l}\text { Tech- } \\
\text { nique }\end{array}$ & $\eta$ \\
\hline \multirow[t]{3}{*}{ Rac DPP } & $t t / g g$ & $\eta^{2} / 1$ & & $\mathrm{DRS}^{2}$ & 1.48 \\
\hline & & & -706.4 & $\mathrm{NMR}^{8}$ & 1.82 \\
\hline & tgtt/gtgg & $\eta^{2} / 1$ & & DRS & 1.48 \\
\hline \multirow[t]{3}{*}{ Hetero TPH } & & & -639.5 & $\mathrm{NMR}^{4}$ & 1.70 \\
\hline & $g t t t / g t g g$ & $\eta^{2} / 1$ & & DRS & 1.48 \\
\hline & & & -586.4 & $\mathrm{NMR}^{4}$ & 1.63 \\
\hline \multirow[t]{2}{*}{ Syndio TPH } & tttt/ttgg & $\eta^{2} / 1$ & & DRS & $1.39^{\mathrm{a}}$ \\
\hline & (or $g g t$ & & -396.7 & $\mathrm{NMR}^{4}$ & 1.40 \\
\hline
\end{tabular}
$\eta$ determined from DRS and NMR

a This value fits exactly the experimental $\left\langle\gamma^{2}\right\rangle$ of this stereoisomer, all things being equal elsewhere. 
formers of model compounds of PS. NMR permits access ${ }^{4}$ to the free enthalpy difference $\Delta G$ between the various molecular conformations of studied compounds and therefore to the statistical weight $\eta$ from the relation ${ }^{10} \eta^{2}=$ $\exp (-\Delta G / R T)$.

In Table II we have indicated for various conformers the statistical weights (which represent also the equilibrium composition), the free enthalpy difference $\Delta G$ between these conformers and the $\eta$ values calculated at $25^{\circ} \mathrm{C}$ from DRS and NMR.

It can be shown that i) first, the $\eta$ values deduced from depolarized Rayleigh scattering measurements at $25^{\circ} \mathrm{C}$ on the different model molecules of PS are homogeneous and in a range of 1.39 to 1.48 and ii) secondly, these values are of the same order of magnitude, within about $10 \%$, as that obtained by $\mathrm{NMR}^{4}$ on $\mathrm{TPH}$ molecules. However, a rather large disagreement of about $20 \%$ was detected between the preceding values and those determined by $\mathrm{NMR}^{8}$ from DPP.

In conclusion it has been possible to give a coherent interpretation, as a whole, on the ex- perimental molecular optical anisotropies of model molecules of PS in good agreement with literature data $^{4,8}$ on statistical weight $\eta$ and results already published on molecular optical anisotropy ${ }^{1,2}$ of PS chains.

\section{REFERENCES}

1. G. Fourche and M. T. Jacq, Polym. J, 4, 465 (1973).

2. G. Fourche and B. Lemaire, Polym. J, 4, 476 (1973).

3. P. Bothorel, J. Colloid. Interface Sci., 27, 529 (1968).

4. H. Pivcova, M. Kolinsky, D. Lim, and B. Schneider, J. Polym. Sci. Part C, 22, 1093 (1969).

5. A. E. Tonelli, Y. Abe, and P. J. Flory, Macromolecules, 3, 803 (1970).

6. P. J. Flory, "Statistical mechanics of chain molecules," Interscience, New York, N.Y., 1969.

7. B. Jasse, Results not published.

8. F. A. Bovey, F. P. Hood, E. W. Anderson, and L. C. Snyder, J. Chem. Phys., 42, 3900 (1965).

9. B. Jasse and L. Monnerie, J. Phys. D, Appl. Phys., 8, 863 (1975).

10. A. D. Williams and P. J. Flory, J. Am. Chem. Soc., 91, 3111 (1969). 